\title{
Oxygen-rich disk in the V778 Cygni system resolved ${ }^{\star}$
}

\author{
R. Szczerba ${ }^{1}$, M. Szymczak ${ }^{2}$, N. Babkovskaia ${ }^{3}$, J. Poutanen $^{3}$, A. M. S. Richards ${ }^{4}$, and M. A. T. Groenewegen ${ }^{5}$ \\ 1 N. Copernicus Astronomical Center, Rabiańska 8, 87-100 Toruń, Poland \\ e-mail: szczerba@ncac.torun.pl \\ 2 Toruń Centre for Astronomy, Nicolaus Copernicus University, Gagarina 11, 87-100 Toruń, Poland \\ Astronomy Division, PO Box 3000, University of Oulu, 90014 Oulu, Finland \\ ${ }^{4}$ Jodrell Bank Observatory, University of Manchester, Macclesfield, Cheshire SK11 9D1, UK \\ 5 Instituut voor Sterrenkunde, PACS-ICC, Celestijnenlaan 200B, 3001 Leuven, Belgium
}

Received 20 December 2004 / Accepted 2 March 2006

\section{ABSTRACT}

\begin{abstract}
Aims. Various scenarios have been proposed to explain the presence of silicate features associated with carbon stars, such as V778 Cyg. We have attempted to constrain these theories by means of mapping water maser emission from V778 Cyg.

Methods. The $22 \mathrm{GHz}$ water maser emission from this star has been mapped using MERLIN with an astrometric accuracy of 25 mas. Results. The spatially- and kinematically-resolved maser complex is displaced by $\sim 190$ mas from the position of the C-star as measured 10 years earlier using Tycho. Our simulations and analysis of available data show that this position difference is unlikely to be due to proper motion if V778 Cyg is at the assumed distance of $1.4 \mathrm{kpc}$. The maser components seem to form a distorted Sshaped structure extended over $\sim 18$ mas with a clear velocity gradient. We propose a model which explains the observed water maser structure as an O-rich warped disk around a companion of the C-star in V 778 Cyg binary system, which is seen almost edge-on. Conclusions. Analysis of observational data, especially those obtained with MERLIN, suggests that V778 Cyg (and, by implication, other silicate carbon stars) are binary systems composed of a C-rich star and a companion which stores circumstellar O-rich material.
\end{abstract}

Key words. stars: AGB and post-AGB - stars: carbon - stars: chemically peculiar - masers

\section{Introduction}

The silicate emission features at about 10 and $18 \mu \mathrm{m}$ are characteristic of O-rich dust envelopes. Surprisingly, these features were also discovered in the IRAS LRS data for some optically classified carbon stars (Little-Marenin 1986; Willems \& de Jong 1986), later termed silicate carbon stars. The detection of silicate emission from these stars suggests that their relatively close surroundings contain oxygen-based dust, in spite of their photospheric chemical composition which shows $\mathrm{C} / \mathrm{O}>1$. An additional argument for the persistence of O-rich material comes from the detection of water and $\mathrm{OH}$ maser lines towards some silicate carbon stars (e.g. Little-Marenin et al. 1994; Engels 1994; Little-Marenin et al. 1988).

Little-Marenin (1986) proposed that silicate carbon stars are binaries consisting of $\mathrm{C}$-rich and $\mathrm{O}$-rich giants, but extensive observations (e.g. Lambert et al. 1990; Engels \& Leinert 1994) did not show any evidence for an O-rich giant in these sources. Willems \& de Jong (1986) proposed that silicate carbon stars were formed very recently due to a thermal pulse which changed the chemistry of the star from O- to C-rich and that the O-rich material ejected before and during the thermal pulse gives the observed silicate features. However, the Infrared Space Observatory (ISO) spectra showed that during the 14-year time interval between IRAS and ISO missions the shape and intensity of the silicate features in V778 Cyg did not change at all (Yamamura et al. 2000). This suggests that O-rich material is located in some stable configuration and that the

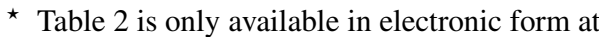
http: //WwW . edpsciences.org model of a fast transition from O- to C-rich star cannot apply, as the silicate features should diminish in strength quite rapidly. Presently, the most widely accepted scenario which is able to explain this phenomenon is a binary system composed of a C-star and an unseen, most likely main-sequence, companion with a reservoir of O-rich material (Morris et al. 1987; Lloyd Evans 1990). Yamamura et al. (2000) argued that the dust responsible for the observed silicate features is stored in a disk around the companion.

To test these hypotheses we observed water masers towards V778 Cyg at high angular resolution. Previous observations only revealed unresolved radio emission within 0.5 of the position of the optical star (Deguchi et al. 1988; Colomer et al. 2000). We also present a simple quantitative interpretation of the observed structure based on a Keplerian disk model.

\section{Observations and data reduction}

The observations were taken on 2001 October 12/13 under good weather conditions, using five telescopes of MERLIN (Diamond et al. 2003). The longest MERLIN baseline of $217 \mathrm{~km}$ gave a fringe spacing of 12 mas at $22 \mathrm{GHz}$. A bandwidth of $2 \mathrm{MHz}$ was used divided into 256 spectral channels per baseline providing a channel separation of $0.105 \mathrm{~km} \mathrm{~s}^{-1}$. The velocities $\left(V_{\mathrm{LSR}}\right)$ were measured with respect to the local standard of rest. The continuum calibrator sources were observed in $16 \mathrm{MHz}$ bandwidth. We used the phase referencing method; 4 min scans on V778 Cyg were interleaved with 2 min scans on the source $2021+614$ (at 3.8 from the target) over $11.5 \mathrm{~h}$. VLBA observations at $8.6 \mathrm{GHz}$ resolve $2021+614$ into two components separated by 7 mas along the position angle of $33^{\circ}$ 
(Fey et al. 1996). We detect only one unresolved source at $22 \mathrm{GHz}$. Its absolute position coincides within less than 2 mas with the VLBA position of the stronger component at 8.6 GHz. The flux density of $2021+614$ of $1.48 \mathrm{Jy}$ was derived from $4 \mathrm{C} 39.25$, which had a flux density of $7.5 \pm 0.3 \mathrm{Jy}$ at the epoch of our observations (Terasranta 2002, private communication). This source was also used for bandpass calibration.

After initial calibration with MERLIN software, the data were processed using the AIPS package (Greisen 1994). To derive phase and amplitude corrections for atmospheric and instrumental effects the phase reference source was mapped and self-calibrated. These corrections were applied to the V778 Cyg visibility data. The absolute position of the brightest feature at $-15.1 \mathrm{~km} \mathrm{~s}^{-1}$ was determined before further calibration. Finally, the clean components of this image were used as a model for phase self-calibration of this channel and the solutions were applied to all channels. Each channel was then mapped and cleaned using a 12 mas circular restoring beam. We present results for total intensity (Stokes $I$ ) images. The map noise of $\sim 27 \mathrm{mJy} \mathrm{beam}^{-1}$ in a line-free channel was close to the predicted thermal noise level.

To determine the position and the brightness of the maser components two dimensional Gaussian components were fitted to the emission in each channel maps. The position uncertainty depends on the channel signal to noise ratio (Condon et al. 1998; Richards et al. 1999, and references therein) and is lower than 1 mas for about $80 \%$ of the maser components towards V778 Cyg. The absolute position of the phase reference source is known within $\sim 3$ mas. The uncertainties in the absolute positions of the maser components are dominated by errors in the telescope positions and tropospheric effects. Uncertainties in telescope positions of $1-2 \mathrm{~cm}$ cause a maser position error of $\sim 10$ mas. The latter uncertainty was estimated by observing the phase rate on the point source 4C 39.25 which appeared to introduce a position error of $\$ 9$ mas. We checked this using reverse phase referencing. Emission from 15 channels around the reference feature at $-15.1 \mathrm{~km} \mathrm{~s}^{-1}$ was averaged and mapped. The map obtained was used as a model to self-calibrate the raw target data and these solutions were then applied to the raw data of $2021+614$. The position of the reference source was shifted by only $\sim 2$ mas with respect to the catalog position. These factors imply that the absolute position accuracy of the maser source is $\$ 25$ mas. All MERLIN coordinates are given in the ICRS system.

\section{Results and discussion}

A single, unresolved maser component brighter than

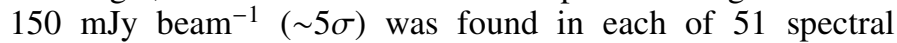
channels. The overall distribution of the $\mathrm{H}_{2} \mathrm{O}$ maser components in V778 Cyg is shown in Fig. 1 (parameters of maser components are listed in Table 2). The total angular extent of the maser emission is about 18 mas. All observed maser components seem to form a distorted S-like shape at a position angle (PA) of about $-10^{\circ}$. However, the most spatially extended series of components $\left(V_{\mathrm{LSR}} \sim-17 \mathrm{~km} \mathrm{~s}^{-1}\right)$ are aligned along $\mathrm{PA} \approx+18^{\circ}$. There is a clear velocity gradient along the whole structure, blue-shifted in the south with respect to the brightest northern components.

The inset in Fig. 1 shows the cross-correlation water maser spectrum towards V778 Cyg. The emission was dominated by a $-15 \mathrm{~km} \mathrm{~s}^{-1}$ feature. Weak emission of about 200-250 mJy was seen at -19 and $-22 \mathrm{~km} \mathrm{~s}^{-1}$. The spectral shape is roughly similar to that of the single dish spectra observed by

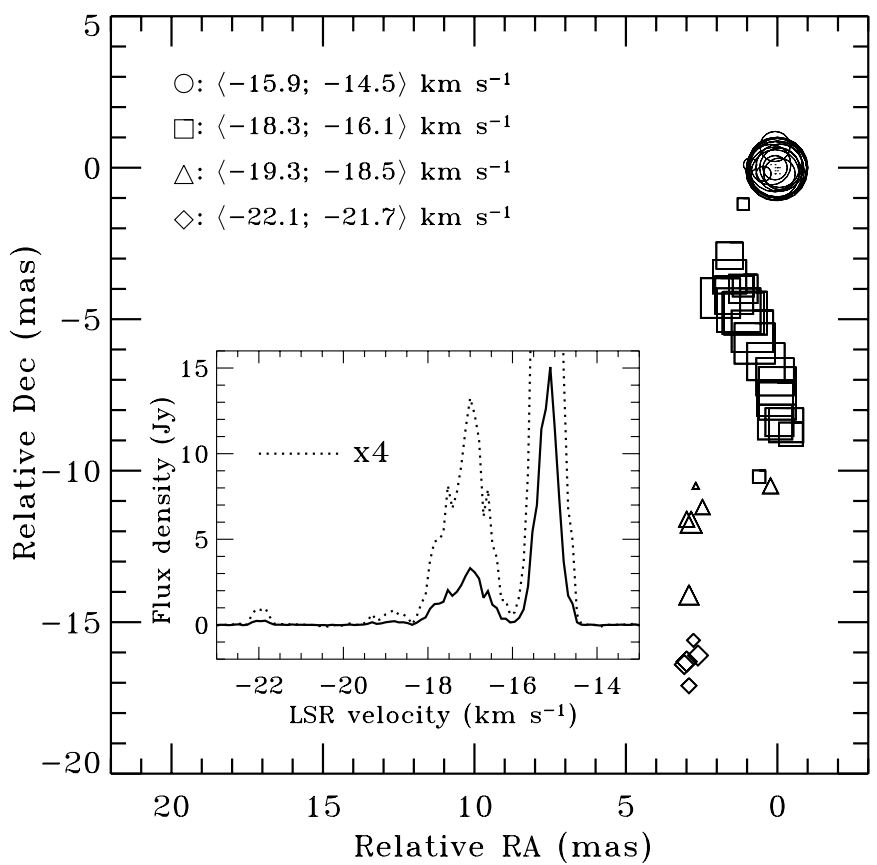

Fig. 1. Positions of the water maser components in V778 Cyg relative to the reference component at $-15.1 \mathrm{~km} \mathrm{~s}^{-1}$. The symbols correspond to the velocity ranges given in the upper left corner. The size of each symbol is proportional to the logarithm of peak brightness of the corresponding component. Inset: MERLIN spectrum of the $\mathrm{H}_{2} \mathrm{O}$ maser emission in V778 Cyg. The dotted line shows the spectrum magnified by a factor of four to enlarge the weak features.

Table 1. Radio and optical coordinates (with errors) for V778 Cyg.

\begin{tabular}{lll}
\hline \hline \multicolumn{2}{c}{ Year RA(J2000) } & Dec(J2000) \\
\hline MERLIN & $200120^{\mathrm{h}} 36^{\mathrm{m}} 07.3833\left( \pm 0^{\mathrm{s}} .0008\right)$ & $60^{\circ} 05^{\prime} 26^{\prime \prime} 024\left( \pm 0^{\prime} .025\right)$ \\
Tycho2 & $199120^{\mathrm{h}} 36^{\mathrm{m}} 07^{\top} .4022( \pm 0.0028)$ & $60^{\circ} 05^{\prime} 26^{\prime \prime} .154\left( \pm 00^{\prime} 040\right)$ \\
\hline
\end{tabular}

Engels \& Leinert (1994) and by Nakada et al. (1987), apart from $\mathrm{a}-22 \mathrm{~km} \mathrm{~s}^{-1}$ feature first detected on 11.3 .95 by Engels ${ }^{1}$.

The absolute position of the reference feature at $-15.1 \mathrm{~km} \mathrm{~s}^{-1}$ is given in Table 1 together with the optical position from the Tycho 2 catalog (Hog et al. 2000). The source is not listed in the Hipparcos catalog. The Tycho2 positions are based on the same observations as the Tychol catalog (ESA 1997) collected by the star mapper of the ESA Hipparcos satellite, but Tycho2 is much bigger and more precise, owing to a more advanced reduction technique. The mean satellite observation epoch is 1991.5. The coordinates given in the Tycho 2 catalog are in the ICRS system and for a star of VT = 10.5 such as V778 Cyg, the estimated astrometric error is at a level of 40 mas. For about 100000 stars (among them for V778 Cyg) no proper motion could be derived.

\subsection{Proper motion and binarity}

The angular separation between the positions of the C-star and the maser reference component (given in Table 1) is 192 mas (142 and 130 mas in RA and Dec, respectively). The observed difference seems to be significant $(\sim 4 \sigma$ above the accuracy level from Table 1 which is about 48 mas), but the epochs of

\footnotetext{
1 wWw.hs.uni-hamburg.de/DE/Ins/Per/Engels/engels/ wcatalog.html
} 
optical and radio observations differ by about 10 years. The C-star would need a proper motion of $\left(\mu_{\alpha} \cos (\mathrm{Dec}), \mu_{\delta}\right)$ close to $(-10,-10)$ mas $\mathrm{yr}^{-1}$ to produce the observed difference in radio and optical positions. Khrutskaya et al. (2004) have estimated the proper motion of V778 Cyg from the difference between the mean position of the star in the two epochs of observations at the Pulkovo Observatory in (1935-1960) and in (1969-1980). The Tycho 2 catalog was used as a reference catalog for astrometric reduction of the Pulkovo plates. Khrutskaya et al. (2004) estimate the proper motion of V778 Cyg to be in the range $\sim[(-8.2 \div$ $-2.3),(-3.9 \div+8.4)]$ mas $\mathrm{yr}^{-1}$, not enough to match optical and radio positions even if observational errors are taken into account.

The problem of proper motion was further investigated using the kinematic model of Groenewegen (2005, in preparation), which includes Galactic rotation. For the present calculations, random motions were drawn from Gaussian velocity ellipsoids with zero mean and dispersions, typical for giants, of 31, 21 and $16 \mathrm{~km} \mathrm{~s}^{-1}$ (Delhaye 1965) in the $U, V, W$ directions, respectively $^{2} .1000$ simulations were performed to calculate proper motions and radial velocities in the direction of V778 Cyg $(l=$ $95^{\circ} .6, b=+11.51$ ) for distances of $0.5,1$ and $2 \mathrm{kpc}$. Figure 2 shows the results producing a $V_{\mathrm{LSR}}$ close to that of $\mathrm{V} 778 \mathrm{Cyg}$, $-20 \pm 5 \mathrm{~km} \mathrm{~s}^{-1}$ (determined from the heliocentric radial velocity of about $-35 \mathrm{~km} \mathrm{~s}^{-1}$, reported by Barbier-Brossat \& Figon 2000). The distribution of points in Fig. 2 demonstrate that it is unlikely that an evolved star with $V_{\mathrm{LSR}}=-20 \pm 5 \mathrm{~km} \mathrm{~s}^{-1}$ (V778 Cyg) at a distance between 0.5 and $2.0 \mathrm{kpc}$ would have a proper motion sufficient to match the optical C-star position with the radio position. This would require a transverse velocity of order of $(-10,-10)$ mas $\mathrm{yr}^{-1}$, shown by the diamond in Fig. 2, which is outside all the predictions of our simulation. Note, that the distance to V778 Cyg is estimated to be $D \simeq 1.4 \mathrm{kpc}$ (Peery 1975; Yamamura et al. 2000).

Therefore, we believe that existing observations and simulations support a binary system model discussed in detail by Yamamura et al. (2000) and that the water maser is associated with a companion star. However, observational errors are not negligible and simultaneous optical and radio observations would be required to confirm this hypothesis firmly.

The water maser components at $-15,-17$ and $-19 \mathrm{~km} \mathrm{~s}^{-1}$ have been detected several times during the last 15 years (Nakada et al. 1987; Engels \& Leinert 1994; this paper). Comparison of these observations show that changes in their radial velocities, $\Delta V$, do not exceed $0.5 \mathrm{~km} \mathrm{~s}^{-1}$. If the velocity change is due to the orbital motion of the secondary (with its maser) around the carbon star (mass $M_{\mathrm{c}}$ ), the rate of change (independently of the companion mass) is given by:

$\frac{\Delta V}{\Delta t} \simeq \frac{G M_{\mathrm{c}}}{d^{2}} \sin i$,

where $\Delta t$ is the time between observations, $G$ is the gravitational constant and $i$ is the inclination angle. Hence, for $i \simeq 90^{\circ}$, the distance $d$ between the binary companions is given by:

$d \gtrsim 75\left(\frac{\Delta V}{0.5 \mathrm{~km} \mathrm{~s}^{-1}}\right)^{-1 / 2}\left(\frac{\Delta t}{15 \text { years }}\right)^{1 / 2}\left(\frac{M_{\mathrm{c}}}{M_{\odot}}\right)^{1 / 2} \mathrm{AU}$.

2 The following parameters also enter the model: a distance SunGalactic Centre of $8.5 \mathrm{kpc}$ (Kerr \& Lynden-Bell 1986), Oort's constants of $A=14.4 \mathrm{~km} \mathrm{~s}^{-1} \mathrm{kpc}^{-1}, B=-12.0 \mathrm{~km} \mathrm{~s}^{-1} \mathrm{kpc}^{-1}$, and higher order terms $\frac{\mathrm{d}^{2} \theta}{\mathrm{d} r^{2} \mid R_{0}}=-3.4 \mathrm{~km} \mathrm{~s}^{-1} \mathrm{kpc}^{-2}, \frac{\mathrm{d}^{3} \theta}{\mathrm{d} r^{3} \mid R_{0}}=2.0 \mathrm{~km} \mathrm{~s}^{-1} \mathrm{kpc}^{-3}$ (Pont et al.1994), and a Solar motion of $19.5 \mathrm{~km} \mathrm{~s}^{-1}$ in the direction $l=56^{\circ} .0$, $b=23^{\circ} .0$ (Feast \& Whitelock 1997).

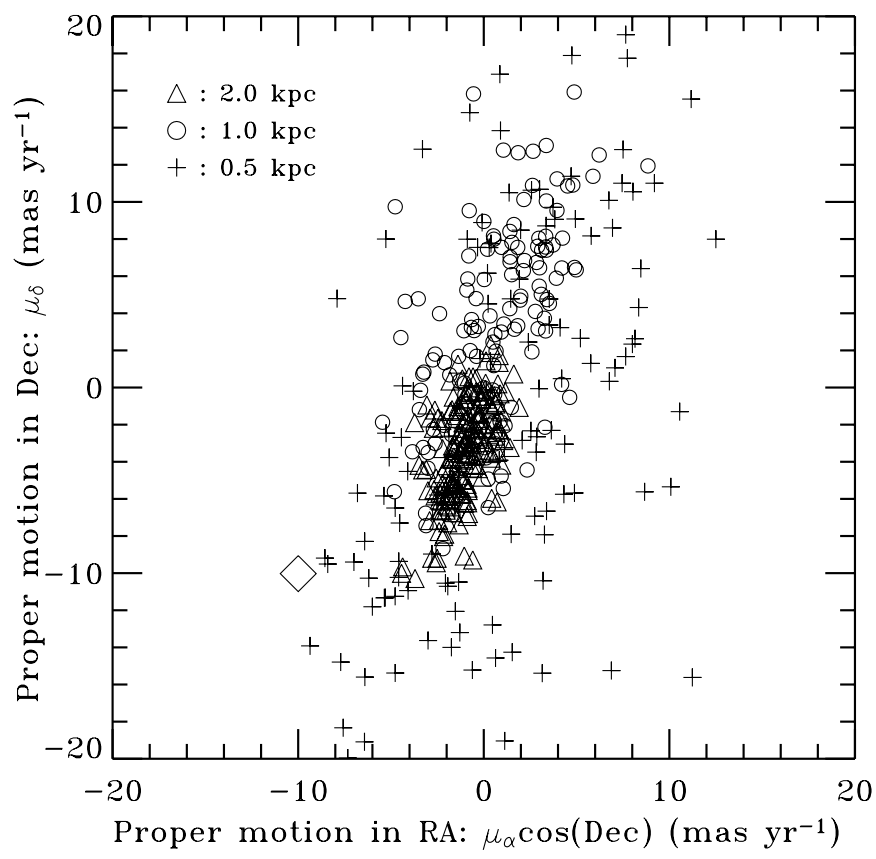

Fig. 2. Different realisations of the proper motions for an evolved star with a LSR velocity close to $-20 \mathrm{~km} \mathrm{~s}^{-1}$, which lies in the direction of V778 Cyg at different distances (see text for details). The proper motion required to match optical $\mathrm{C}$-star and radio maser position is marked by diamond.

\subsection{Where do the water masers come from?}

The distorted S-shaped structure seen in Fig. 1 can be interpreted as a warped disk which may be non-co-planar with the orbital plane and is seen almost edge-on. The disk might be made up of clumps which share the disk's rotation. Tidal forces (especially in a non-co-planar disk) could produce shear and/or density waves (e.g. Papaloizou \& Terquem 1995) responsible for the observed distortion. We assume that the disk is at $\mathrm{PA}=+18^{\circ}$, as suggested by the elongation angle of emission around the central $V_{\mathrm{LSR}} \sim-17 \mathrm{~km} \mathrm{~s}^{-1}$, and show the assumed orientation by the straight solid line in Fig. 3. The shaded delimiting triangle, in this figure, marks the allowed C-star locations at the epoch of our observations (2001.78) as inferred from the proper motion estimations by Khrutskaya et al. (2004). The delimiting triangle has one corner at the optical C-star position (no proper motion at all) and the two others determined by the extreme limits to proper motion $\left(\mu_{\alpha} \cos (\mathrm{Dec}), \mu_{\delta}\right)$ from Khrutskaya et al. (2004). The lower limit is derived from $(-8.2,-3.9)$ and the upper (off the top of the plot) is derived from $(-2.3,+8.4)$ mas $_{\mathrm{yr}^{-1}}$. The allowed $\mathrm{C}$-star locations determine also the allowed positions (at the time of MERLIN observations) of the orbital plane of the system. The position of orbital plane in case of no proper motion is shown by the dotted line in Fig. 3.

From Fig. 3 it is seen that the proposed interpretation is consistent with the observational constraints and the disk may be slightly (or may not be, if observational errors are taken into account) tilted relative to the orbital plane. Note that assuming (as suggested by the overall structure seen in Fig. 1) that the disk plane lies at position angle of $-10^{\circ}$ (dashed line in Fig. 3) we would not be able to fulfill constraints imposed by the proper motion estimation of V778 Cyg (shaded region in Fig. 3) by Khrutskaya et al. (2004), unless the disk is tilted by more than about $30^{\circ}$. 


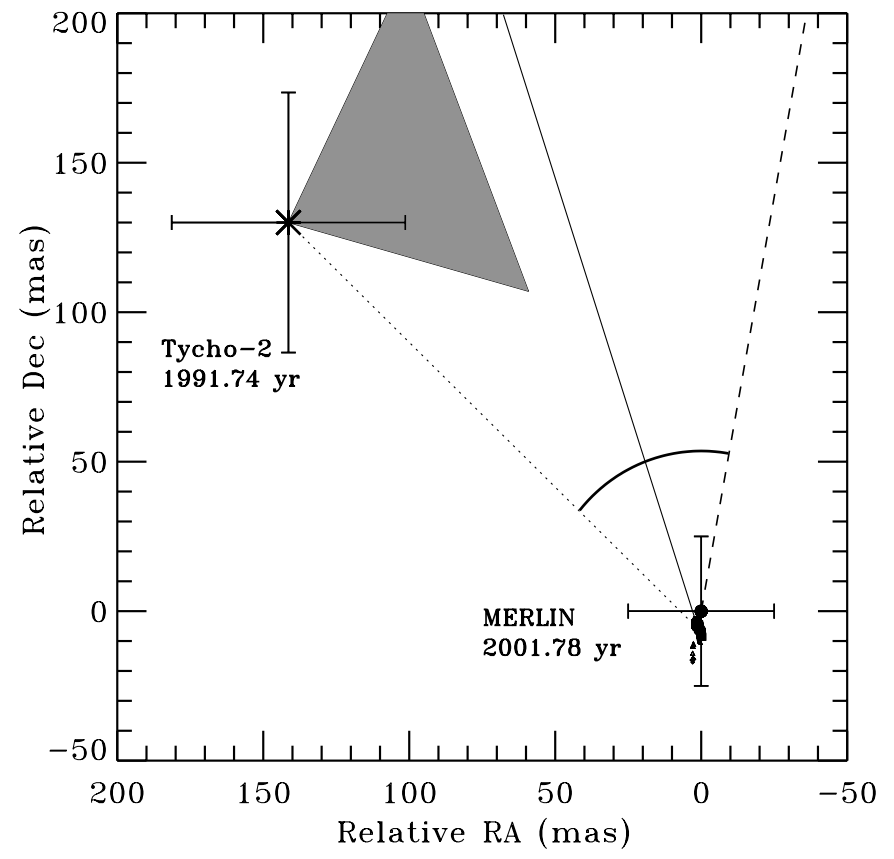

Fig. 3. Relative positions (together with errors) of the C-star and water maser in V778 Cyg. The assumed disk orientation is shown by the straight solid line. The shaded region marks the allowed C-star locations which are inferred from the proper motion estimations (see text for details). The sector of a circle marks the minimal allowed angular distance of the C-star from the radio MERLIN position (as suggested by Eq. (2)) assuming that the distance to V778 Cyg is $1.4 \mathrm{kpc}$. The straight dotted line shows the position of orbital plane in case of no proper motion, while the dashed line marks the position angle of the overall structure seen in Fig. 1.

The above interpretation is strengthened by Fig. 4, which shows the $V_{\mathrm{LSR}}$ of the water maser components versus major axis offset $x,(V-x$ diagram). The major axis is assumed to be at $\mathrm{PA}=+18^{\circ}$ as suggested by the elongation angle of emission around the central $V_{\mathrm{LSR}} \sim-17 \mathrm{~km} \mathrm{~s}^{-1}$. Note, that we only detect one unresolved maser component per channel and therefore the apparent position of the components is along the line of sight of the greatest amplification at the $V_{\mathrm{LSR}}$ sampled by that channel. Figure 4 shows an almost linear velocity gradient with a greater velocity range per unit distance at the extremes. This is characteristic of emission from an almost edgeon disk in Keplerian rotation (e.g. Shepherd \& Kurtz 1999). As unsaturated maser amplification is exponential, a relatively small change in optical depth arising from a differential velocity gradient produces strong domination by emission at the favored velocity (derived rigorously in Pestalozzi et al. 2004, and references therein). In contrast, water masers associated with evolved-star jets (Miranda et al. 2001; Imai et al. 2002) typically show more fan-like structures with a less ordered velocity gradient which tends to be steepest in the centre, not at the limbs. Note also that the observed radial velocity gradient (about $7 \mathrm{~km} \mathrm{~s}^{-1}$ in $\sim 17$ mas, i.e. about $0.3 \mathrm{~km} \mathrm{~s}^{-1} \mathrm{AU}^{-1}$ at distance of $1.4 \mathrm{kpc}$ ) seems to be too small for typical jets. In addition, the stability of the maser velocities on the scale of 15 years (see Sect. 3.1) suggests also that the maser originate from a stable configuration, but not from an outflow or jet. Therefore we develop our discussion in the context of a disk model only. Multi-epoch (proper motion) observations are needed to rule out a jet completely.

Assuming that the disk is in Keplerian rotation and that the LSR velocity of the disk center $\left(V_{\mathrm{LSR}}^{\mathrm{dc}}\right)$ is about $-17 \mathrm{~km} \mathrm{~s}^{-1}$,

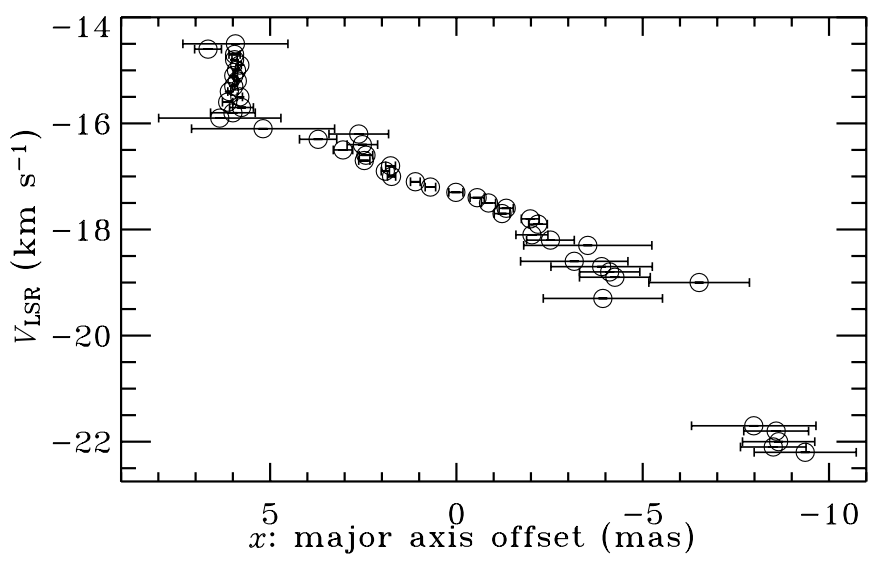

Fig. 4. The LSR velocity against the distance along the major axis for water maser components in V778 Cyg. The major axis is assumed to be a line at $\mathrm{PA}=+18^{\circ}$ defined by the $V_{\mathrm{LSR}} \sim-17 \mathrm{~km} \mathrm{~s}^{-1}$ water maser components; the zero of angular offset marks the centre of this structure.

we can express the line of sight velocity at impact parameter $x$ (major axis offset) as $V_{x}=V_{\mathrm{LSR}}^{\mathrm{dc}}+V_{\mathrm{K}}(x / R) \mathrm{km} \mathrm{s}^{-1}$, where $V_{\mathrm{K}}$ is the assumed Keplerian velocity at the disk edge of radius $R$. The emission from an edge-on Keplerian disk has three maxima: one corresponding to the systemic velocity (here about $-17 \mathrm{~km} \mathrm{~s}^{-1}$ ) and associated with matter on the line of sight towards the disk centre (as modelled by Pestalozzi et al. 2004), and two components (here, -15 and $-19 \mathrm{~km} \mathrm{~s}^{-1}$ ) associated with the outer edges of the disk where emission peaks close to the Keplerian rotation velocity (see e.g. Grinin \& Grigor'ev 1983; Watson \& Wyld 2000). For a Keplerian velocity of $2 \mathrm{~km} \mathrm{~s}^{-1}$, the radius of the disk $R$ is about $1 / 3$ larger than the impact parameter at which the amplification is maximal $x_{\max } \simeq 7$ mas. This results in $R \simeq(4 / 3) \times 7$ mas $\times D[\mathrm{kpc}]=13 \mathrm{AU}(D / 1.4) \mathrm{kpc}$. Assuming that the mass of the disk itself is negligible in comparison with mass of the secondary star, we can derive the central mass inside the disk using Kepler's laws, giving $M_{\mathrm{s}} \simeq 0.06(D / 1.4 \mathrm{kpc}) M_{\odot}$. Note, that an object of such a small mass (brown dwarf?) would not be detected by speckle interferometry as performed by Engels \& Leinert (1994).

The proposed model can easily explain components at -15 , -17 and $-19 \mathrm{~km} \mathrm{~s}^{-1}$. The variations in their relative strengths could be due to changes in physical conditions inside the disk: e.g. radial temperature gradient (the temperature should be highest at the northern disk edge), sub-sonic turbulence or clumpiness. However, this model cannot explain the weakest component at $-22 \mathrm{~km} \mathrm{~s}^{-1}$. It is possible that this feature is formed in material evaporating from the disk (see model of Yamamura et al. 2000). On the other hand, non-detection of an edge of the disk is also possible. As exponential, directional maser amplification exaggerates underlying conditions, some asymmetry is not surprising: there could even be material at corresponding red-shifted velocities where masing is temporarily disrupted. If we do not detect the extremities of the disc, or if the angle of inclination is not quite $90^{\circ}$, the central star could be more massive (although still sub-Solar). Finally, note that the observed strength of the water maser could be explained for the water concentration of about $10^{-5} \mathrm{~cm}^{-3}$ and the gas temperature in the disk of about $300 \mathrm{~K}$. Such a temperature is possible to achieve by the heating of dust inside the disk by the radiation from the C-star and the energy exchange between dust and gas (Babkovskaia et al. in preparation). 


\section{Conclusions}

We mapped the water maser emission from silicate carbon star V778 Cyg using MERLIN. The radio position obtained from MERLIN in 2001 is at an angular separation of 190 mas from the optical position of V778 Cyg given in the 1991 Tycho 2 catalog. This cannot be explained by proper motion if V778 Cyg is at a distance of $\sim 1.4 \mathrm{kpc}$ and instead (probably) provides observational support for the binary system model of Yamamura et al. (2000). Simultaneous radio and optical measurements are needed to verify this model which will be developed in a further paper. The velocity changes of the main maser components over 15 years imply that the distance between the C-star and disk is at least $75 \mathrm{AU}$. The water maser components have an almost linear, S-shaped distribution as projected on the sky and in the variations of $V_{\mathrm{LSR}}$ along the elongation of the main component at about $-17 \mathrm{~km} \mathrm{~s}^{-1}$. We suggest that this can be interpreted as an almost edge-on warped Keplerian disk located around a companion object and tilted by no more than $20^{\circ}$ relative to the orbital plane. We estimate that the central mass inside the disk is $\gtrsim 0.06 M_{\odot}$ (for a distance of $1.4 \mathrm{kpc}$ ).

Acknowledgements. This work has been supported by grant 2.P03D 017.25 of the Polish State Committee for Scientific Research (RS), the Magnus Ehrnrooth Foundation, and the Finnish Graduate School for Astronomy and Space Physics (NB), and the Academy of Finland (JP).

\section{References}

Barbier-Brossat, M., \& Figon, P. 2000, A\&AS, 142, 217

Colomer, F., Reid, M. J., Menten, K. M., \& Bujarrabal, V. 2000, A\&A, 355, 979

Condon, J. J., Cotton, W. D., Greisen, E. W., et al. 1998, AJ, 115, 1693
Deguchi, S., Kawabe, R., Ukita, N., et al. 1988, ApJ, 325, 795

Delhaye, J. 1965, in Galactic structure, Stars and Stellar systems, 5, ed. A. Blaauw, \& M. Schmidt (University of Chicago Press)

Diamond, P. J., Garrington, S. T., Gunn, A. G., et al. 2003, MERLIN User Guide, ver. 3

Engels, D. 1994, A\&A, 285, 497

Engels, D., \& Leinert, Ch. 1994, A\&A, 282, 858

ESA 1997, The Hipparcos and Tycho Catalogues, ESA SP-1200

Feast, M., \& Whitelock, P. 1997, MNRAS, 291, 683

Fey, A. L., Clegg, A. W., \& Fomalont, E. B. 1996, ApJS, 105, 299

Greisen, E. W. 1994, AIPS Cookbook, NRAO

Grinin, V. P., \& Grigor'ev, S. A. 1983, Pis'ma Astron. Zh., 9, 463

Hog, E., Fabricius, C., Makarov, V. V., et al. 2000, A\&A, 355, L27

Imai, H., Obara, K., Diamond, P. J., Omodaka, T., \& Sasao, T. 2002, Nature, 417, 829

Kerr, F. J., \& Lynden-Bell, D. 1986, MNRAS, 221, 1023

Khrutskaya, E. V., Khovritchev, M. Y., \& Bronnikova, N. M. 2004, A\&A, 418, 357

Lambert, D. L., Hinkle, K. H., \& Smith, V. V. 1990, AJ, 99, 1612

Little-Marenin, I. R. 1986, A\&A, 307, L15

Little-Marenin, I. R., Benson, P. J., \& Dickinson, D. F. 1988, ApJ, 330, 828

Little-Marenin, I. R., Sahai, R., Wannier, P. G., et al. 1994, A\&A, 281, 451

Lloyd Evans, T. 1990, MNRAS, 243, 336

Miranda, L. F., Gómez, Y., Anglada, G., \& Torrelles, J. M. 2001, Nature, 414, 284

Morris, M., Guilloteau, S., Lucas, R., \& Omont, A. 1987, ApJ, 321, 888

Nakada, Y., Izumiura, H., Onaka, T., et al. 1987, ApJ, 323, L77

Papaloizou, J. C. B., \& Terquem, C. 1995, MNRAS, 274, 987

Peery, B. F., Jr. 1975, ApJ, 199, 135

Pestalozzi, M. R., Elitzur, M., Conway, J. E., \& Booth, R. S. 2004, ApJ, 603, L113

Pont, F., Mayor, M., \& Burki, G. 1994, A\&A, 285, 415

Richards, A. M. S., Yates, J. A., \& Cohen, R. J. 1999, MNRAS, 306, 954

Shepherd, D. S., \& Kurtz, S. E. 1999, ApJ, 523, 690

Watson, W. D., \& Wyld, H. W. 2000, ApJ, 530, 207

Willems, F. J., \& de Jong, T. 1986, ApJ, 309, L39

Yamamura, I., Dominik, C., de Jong, T., et al. 2000, A\&A, 363, 629 


\section{Online Material}


R. Szczerba et al.: O-rich disk in V778 Cyg, Online Material p 2

Table 2. Parameters of water maser components from MERLIN observations of V778 Cyg.

\begin{tabular}{|c|c|c|c|c|c|c|}
\hline Channel & $V_{\mathrm{LSR}}\left(\mathrm{km} \mathrm{s}^{-1}\right)$ & $\mathrm{RA}(\mathrm{J} 2000)$ & $\operatorname{Dec}(J 2000)$ & Flux (Jy/beam) & Flux error & Position error (mas) \\
\hline 103 & -14.5 & 203607.38332 & 600526.0238 & $3.8470 \mathrm{E}-01$ & $2.60 \mathrm{E}-02$ & 1.41 \\
\hline 104 & -14.6 & 203607.38327 & 600526.0247 & $1.5015 \mathrm{E}+00$ & $2.61 \mathrm{E}-02$ & 0.36 \\
\hline 105 & -14.7 & 203607.38325 & 600526.0240 & $3.5478 \mathrm{E}+00$ & $2.68 \mathrm{E}-02$ & 0.15 \\
\hline 106 & -14.8 & 203607.38325 & 600526.0240 & $7.1388 \mathrm{E}+00$ & $2.57 \mathrm{E}-02$ & 0.07 \\
\hline 107 & -14.9 & 203607.38327 & 600526.0238 & $1.2418 \mathrm{E}+01$ & $2.89 \mathrm{E}-02$ & 0.04 \\
\hline 108 & -15.0 & 203607.38327 & 600526.0239 & $1.9127 \mathrm{E}+01$ & $3.18 \mathrm{E}-02$ & 0.03 \\
\hline 109 & -15.1 & 203607.38326 & 600526.0240 & $2.4397 \mathrm{E}+01$ & $2.49 \mathrm{E}-02$ & 0.02 \\
\hline 110 & -15.2 & 203607.38326 & 600526.0239 & $2.1525 \mathrm{E}+01$ & $3.30 \mathrm{E}-02$ & 0.02 \\
\hline 111 & -15.3 & 203607.38326 & 600526.0240 & $1.9169 \mathrm{E}+01$ & $3.06 \mathrm{E}-02$ & 0.03 \\
\hline 112 & -15.4 & 203607.38327 & 600526.0241 & $1.3279 \mathrm{E}+01$ & $2.93 \mathrm{E}-02$ & 0.04 \\
\hline 113 & -15.5 & 203607.38327 & 600526.0238 & $7.6574 \mathrm{E}+00$ & $2.69 \mathrm{E}-02$ & 0.07 \\
\hline 114 & -15.6 & 203607.38329 & 600526.0241 & $3.9029 \mathrm{E}+00$ & $2.63 \mathrm{E}-02$ & 0.14 \\
\hline 115 & -15.7 & 203607.38325 & 600526.0238 & $1.7063 \mathrm{E}+00$ & $2.60 \mathrm{E}-02$ & 0.32 \\
\hline 116 & -15.8 & 203607.38327 & 600526.0240 & $8.9236 \mathrm{E}-01$ & $2.63 \mathrm{E}-02$ & 0.60 \\
\hline 117 & -15.9 & 203607.38338 & 600526.0241 & $3.3061 \mathrm{E}-01$ & $2.68 \mathrm{E}-02$ & 1.64 \\
\hline 118 & -16.1 & 203607.38341 & 600526.0228 & $2.8095 \mathrm{E}-01$ & $2.54 \mathrm{E}-02$ & 1.92 \\
\hline 119 & -16.2 & 203607.38341 & 600526.0201 & $6.7754 \mathrm{E}-01$ & $2.68 \mathrm{E}-02$ & 0.80 \\
\hline 120 & -16.3 & 203607.38347 & 600526.0211 & $1.0845 \mathrm{E}+00$ & $2.63 \mathrm{E}-02$ & 0.50 \\
\hline 121 & -16.4 & 203607.38341 & 600526.0200 & $1.3044 \mathrm{E}+00$ & $2.63 \mathrm{E}-02$ & 0.41 \\
\hline 122 & -16.5 & 203607.38347 & 600526.0204 & $2.0243 \mathrm{E}+00$ & $2.56 \mathrm{E}-02$ & 0.26 \\
\hline 123 & -16.6 & 203607.38345 & 600526.0198 & $3.0483 \mathrm{E}+00$ & $2.69 \mathrm{E}-02$ & 0.18 \\
\hline 124 & -16.7 & 203607.38351 & 600526.0197 & $3.4839 \mathrm{E}+00$ & $2.54 \mathrm{E}-02$ & 0.15 \\
\hline 125 & -16.8 & 203607.38341 & 600526.0192 & $4.2444 \mathrm{E}+00$ & $2.63 \mathrm{E}-02$ & 0.13 \\
\hline 126 & -16.9 & 203607.38343 & 600526.0193 & $5.0099 \mathrm{E}+00$ & $2.68 \mathrm{E}-02$ & 0.11 \\
\hline 127 & -17.0 & 203607.38340 & 600526.0192 & $4.7547 \mathrm{E}+00$ & $2.67 \mathrm{E}-02$ & 0.11 \\
\hline 128 & -17.1 & 203607.38337 & 600526.0186 & $4.2378 \mathrm{E}+00$ & $2.67 \mathrm{E}-02$ & 0.13 \\
\hline 129 & -17.2 & 203607.38336 & 600526.0182 & $3.7844 \mathrm{E}+00$ & $2.73 \mathrm{E}-02$ & 0.14 \\
\hline 130 & -17.3 & 203607.38331 & 600526.0176 & $2.8783 \mathrm{E}+00$ & $2.62 \mathrm{E}-02$ & 0.19 \\
\hline 131 & -17.4 & 203607.38327 & 600526.0171 & $2.9090 \mathrm{E}+00$ & $2.51 \mathrm{E}-02$ & 0.19 \\
\hline 132 & -17.5 & 203607.38326 & 600526.0168 & $2.8753 \mathrm{E}+00$ & $2.66 \mathrm{E}-02$ & 0.19 \\
\hline 133 & -17.6 & 203607.38326 & 600526.0163 & $2.8295 \mathrm{E}+00$ & $2.56 \mathrm{E}-02$ & 0.19 \\
\hline 134 & -17.7 & 203607.38327 & 600526.0164 & $2.4962 \mathrm{E}+00$ & $2.68 \mathrm{E}-02$ & 0.21 \\
\hline 135 & -17.8 & 203607.38327 & 600526.0156 & $2.2390 \mathrm{E}+00$ & $2.64 \mathrm{E}-02$ & 0.24 \\
\hline 136 & -17.9 & 203607.38322 & 600526.0155 & $2.1629 \mathrm{E}+00$ & $2.66 \mathrm{E}-02$ & 0.25 \\
\hline 137 & -18.1 & 203607.38325 & 600526.0156 & $1.2451 \mathrm{E}+00$ & $2.65 \mathrm{E}-02$ & 0.43 \\
\hline 138 & -18.2 & 203607.38320 & 600526.0152 & $8.3832 \mathrm{E}-01$ & $2.59 \mathrm{E}-02$ & 0.64 \\
\hline 139 & -18.3 & 203607.38334 & 600526.0138 & $3.1351 \mathrm{E}-01$ & $2.65 \mathrm{E}-02$ & 1.72 \\
\hline 141 & -18.5 & 203607.38328 & 600526.0156 & $1.7954 \mathrm{E}-01$ & $2.60 \mathrm{E}-02$ & 3.02 \\
\hline 142 & -18.6 & 203607.38362 & 600526.0135 & $3.7605 \mathrm{E}-01$ & $2.62 \mathrm{E}-02$ & 1.44 \\
\hline 143 & -18.7 & 203607.38359 & 600526.0128 & $3.9566 \mathrm{E}-01$ & $2.65 \mathrm{E}-02$ & 1.36 \\
\hline 144 & -18.8 & 203607.38366 & 600526.0124 & $6.6483 \mathrm{E}-01$ & $2.58 \mathrm{E}-02$ & 0.81 \\
\hline 145 & -18.9 & 203607.38364 & 600526.0123 & $5.6821 \mathrm{E}-01$ & $2.55 \mathrm{E}-02$ & 0.95 \\
\hline 146 & -19.0 & 203607.38365 & 600526.0099 & $4.0059 \mathrm{E}-01$ & $2.64 \mathrm{E}-02$ & 1.35 \\
\hline 147 & -19.1 & 203607.38363 & 600526.0115 & $2.1827 \mathrm{E}-01$ & $2.58 \mathrm{E}-02$ & 2.47 \\
\hline 148 & -19.2 & 203607.38317 & 600526.0121 & $1.5076 \mathrm{E}-01$ & $2.61 \mathrm{E}-02$ & 3.60 \\
\hline 149 & -19.3 & 203607.38329 & 600526.0135 & $3.2427 \mathrm{E}-01$ & $2.55 \mathrm{E}-02$ & 1.66 \\
\hline 172 & -21.7 & 203607.38363 & 600526.0084 & $3.2280 \mathrm{E}-01$ & $2.50 \mathrm{E}-02$ & 1.67 \\
\hline 173 & -21.8 & 203607.38366 & 600526.0077 & $6.2120 \mathrm{E}-01$ & $2.66 \mathrm{E}-02$ & 0.87 \\
\hline 174 & -22.0 & 203607.38367 & 600526.0076 & $5.5633 \mathrm{E}-01$ & $2.70 \mathrm{E}-02$ & 0.97 \\
\hline 175 & -22.1 & 203607.38361 & 600526.0079 & $6.0993 \mathrm{E}-01$ & $2.56 \mathrm{E}-02$ & 0.88 \\
\hline 176 & -22.2 & 203607.38365 & 600526.0069 & $3.9179 \mathrm{E}-01$ & $2.70 \mathrm{E}-02$ & 1.37 \\
\hline
\end{tabular}

The table provides the spectral channel number (Col. 1), the velocity (in respect to the local standard of rest) of the detected water maser components (Col. 2), the absolute coordinates (RA and Dec at J2000) of each feature (Cols. 3 and 4, respectively), flux of each maser component and the corresponding flux error (Cols. 5 and 6, respectively), and the position uncertainty of each component (Col. 7). 